\title{
Prognostic factors in hepatocellular carcinoma patients with bone metastases
}

\author{
Sungmin Kim¹, Youngmin Choi', Dong-Won Kwak', Hyung Sik Lee ${ }^{1}$, Won-Joo Hur ${ }^{1}$, Yang Hyun Baek², \\ Sung Wook Lee ${ }^{2}$ \\ 'Department of Radiation Oncology, Dong-A University Hospital, Dong-A University College of Medicine, Busan, Korea \\ ${ }^{2}$ Department of Internal Medicine, Dong-A University Hospital, Dong-A University College of Medicine, Busan, Korea
}

Purpose: To identify the prognostic factors that could influence survival and to compare prognoses of the patients with the number of the risk factors that might assist in the adequate management of hepatocellular carcinoma (HCC) patients with bone metastases that showed a heterogeneous range of survival.

Materials and Methods: A total of 41 patients, treated with radiotherapy (RT) for bone metastases from HCC from 2014 to 2017, were enrolled retrospectively. Survival was determined by the Kaplan-Meier method from the start of the RT for metastatic bone lesions. Pre-RT clinical features were evaluated and their influences on survival were analyzed. The significant factors were considered to compare survivals according to the number of prognostic factors.

Results: Median follow-up was 6.0 months (range, 0.5 to 47.0 months). The median overall survival was 6.5 months, and the 1 -year and 2-year survival rates were $35.5 \%$ and $13.5 \%$, respectively. Multivariate analysis revealed that the Child-Pugh class A group, alpha-fetoprotein increased more than $30 \mathrm{ng} / \mathrm{mL}$, and $\mathrm{HCC}$ size of more than $5 \mathrm{~cm}$ were associated with worse overall survival. The median survivals in HCC with none, 1, 2, and 3 of the aforementioned risk factors were 19.5, 9.0, 2.5, and 1.0 months, respectively $(p<0.05)$.

Conclusion: Our results show that the overall survivals were significantly different according to the number of the risk factors among HCC patients with bone metastases who showed various lengths of survival.

Keywords: Hepatocellular carcinoma, Bone metastasis, Prognosis, Triage, Radiotherapy

\section{Introduction}

Hepatocellular carcinoma (HCC) is the sixth most prevalent cancer and second leading cause of cancer death in South Korea [1]. With advancements in both the diagnosis and treatment of $\mathrm{HCC}$, the median survival times improved markedly from 17.2 months in 2003-2005 to 28.4 months in
2008-2010 [2].

In the past, the survival of HCC was too short to consider metastases as a problem in patients. Before the 20th century, bone metastasis from HCC was rarely reported; for example, only a $0 \%-5 \%$ incidence was reported in a previous study performed in the 1980s [3]. However, the diagnosis of bone metastases has increased recently due to the prolonged

Received 28 February 2019, Revised 03 April 2019, Accepted 22 July 2019.

Correspondence: Youngmin Choi, Department of Radiation Oncology, Dong-A University Hospital, Dong-A University College of Medicine, 26 Daesingongwon-ro, Seo-gu, Busan 49201, Korea. Tel: +82-51-240-5380, E-mail: cymin00@dau.ac.kr (http://orcid.org/0000-0003-0190-4960)

(c) This is an Open Access article distributed under the terms of the Creative Commons Attribution Non-Commercial License (http://creativecommons.org/ licenses/by-nc/4.0/) which permits unrestricted non-commercial use, distribution, and reproduction in any medium, provided the original work is properly cited.

www.e-roj.org 
survival of HCC patients and the advancements in bone imaging techniques $[4,5]$. Bone is now one of the major metastatic sites and accounts for approximately $25.5 \%$ to $38.5 \%$ of the extrahepatic metastases from HCC [6-10].

Bone metastases from HCC are highly vascularized lesions that can cause severe pain and structural and functional destruction, such as pathologic fractures and neurologic deficits. As a result, there is an enormous deterioration of the patients' quality of life (0OL) [11]. Radiation therapy (RT) is the widely accepted and preferred treatment modality for metastatic bone lesions, potentially offering symptomatic palliation of painful sites. Symptom relief following RT was obtained in approximately 60\% to 95\% of the patients and a complete pain response in up to $32 \%$ [12-15]. Furthermore, prevention of pathologic fractures and neurological sequelae could be achieved by RT [16].

Recently, physicians have more frequently encountered HCC patients with bone metastases who have varying lengths of survival. Median overall survivals were reported from 3 to 11 months $[11,13,14]$, and the prognostic factors were heterogeneous $[6,11,13-15,17-19]$.

In this aspect, the aim of this study was to identify the prognostic factors that could affect survival in HCC patients with metastatic bone lesions receiving radiation therapy. Additionally, we tried to compare survivals of the patients according to the number of the risk factors.

\section{Materials and Methods}

A total of 41 patients receiving RT for metastatic bone lesions from HCC between January 2014 and June 2017 were analyzed retrospectively (Table 1). All medical records of each patient were reviewed after the approval of the Institutional Review Board of Dong-A University Hospital (No. DAUHIRB-18-220).

\section{Clinical evaluation}

Metastatic bone lesions were diagnosed by radiological imaging studies, such as computed tomography, magnetic resonance imaging, whole body bone scintigraphy, or positron emission tomography. A metastatic bone lesion with an expansile soft tissue mass observed outside the bone boundary was considered as an extra-osseous extension.

Pretreatment evaluation was performed at the start of the RT, including medical history taking, physical examination, liver function test, serum alpha-fetoprotein (AFP) level (0-15 $\mathrm{ng} / \mathrm{mL}$ ) evaluation, and imaging studies. The liver function of the patients was assessed with Child-Pugh (CP) classification.
Table 1. Patients' characteristics

\begin{tabular}{|c|c|}
\hline Characteristic & Value \\
\hline Age (yr) & $63(42-82)$ \\
\hline$\leq 60$ & $21(51.2)$ \\
\hline$>60$ & $20(48.8)$ \\
\hline \multicolumn{2}{|l|}{ Sex } \\
\hline Male & $35(85.4)$ \\
\hline Female & $6(14.6)$ \\
\hline \multicolumn{2}{|l|}{ ECOG performance status } \\
\hline $0-1$ & $19(46.3)$ \\
\hline $2-4$ & $22(53.7)$ \\
\hline \multicolumn{2}{|l|}{ Child-Pugh classification } \\
\hline A & $32(78)$ \\
\hline$B, C$ & $9(22)$ \\
\hline \multicolumn{2}{|l|}{ AFP level (ng/mL) } \\
\hline$\leq 30$ & $16(39)$ \\
\hline$>30$ & $25(61)$ \\
\hline $\operatorname{ALP}(U / L)$ & $344(71-859)$ \\
\hline Normal & $20(48.8)$ \\
\hline Elevated & $21(51.2)$ \\
\hline GGT (U/L) & $81(16-993)$ \\
\hline Normal & $16(39)$ \\
\hline Elevated & $25(61)$ \\
\hline Primary tumor size $(\mathrm{cm})$ & $4.9(0-14.9)$ \\
\hline$\leq 5$ & $21(51.2)$ \\
\hline$>5$ & $20(48.8)$ \\
\hline \multicolumn{2}{|c|}{ Primary tumor control status ${ }^{a)}$} \\
\hline$C R, P R, S D$ & $19(46.3)$ \\
\hline PD & $17(41.5)$ \\
\hline Unknown & $5(12.2)$ \\
\hline \multicolumn{2}{|l|}{ PVTT } \\
\hline Yes & $11(26.8)$ \\
\hline No & $30(73.2)$ \\
\hline \multicolumn{2}{|l|}{ Extra-osseous extension } \\
\hline Yes & $32(78)$ \\
\hline No & $9(22)$ \\
\hline \multicolumn{2}{|l|}{ No. of bone metastasis } \\
\hline Single & 15 (36.6) \\
\hline Multiple & $26(63.4)$ \\
\hline \multicolumn{2}{|l|}{ Other metastases site } \\
\hline Yes & $18(43.9)$ \\
\hline No & $23(56.1)$ \\
\hline
\end{tabular}

Values are presented as mean \pm standard deviation or number (\%). RT, radiotherapy; ECOG, Eastern Cooperative Oncology Group; AFP, alpha-fetoprotein; ALP, alkaline phosphatase; GGT, gammaglutamyltransferase; $C R$, complete response; $P R$, partial response; $S D$, stable disease; $P D$, progressive disease; $P V T$, portal vein tumor thrombosis.

${ }^{a}$ Response evaluation was conducted according to the modified Response Evaluation Criteria in Solid Tumors guideline. 
Some clinical features were categorized and summarized using frequencies and percentages (Table 1): performance status of Eastern Cooperative Oncology Group (ECOG) 0 and 1 vs. ECOG 2 to 4 ; CP class A vs. CP class B and C; controlled primary tumor vs. progression by modified Response Evaluation Criteria in Solid Tumor (RECIST) $[20,21]$; and less than $30 \mathrm{ng} / \mathrm{mL}$ of serum AFP level vs. more than $30 \mathrm{ng} / \mathrm{mL}$.

\section{Radiotherapy}

Palliative RT was applied in cases of metastatic bone lesions of spinal cord compression, pain affecting the patients' $00 \mathrm{~L}$, high risk of pathologic fractures, and weight-bearing sites without symptoms.

Gross tumor volume (GTV) was contoured as the enhancing mass and osteolytic or osteoblastic changes of the bone in planning CT or diagnostic images. For hypofractionated RT, clinical target volume (CTV) included GTV plus a $2-3 \mathrm{~cm}$ margin longitudinally and $1 \mathrm{~cm}$ axially in long bones and 1-2 $\mathrm{cm}$ in all directions in the other bones. Planning target volume (PTV) was formed by expanding 7-10 mm from the CTV. For stereotactic body radiotherapy (SBRT), CTV included the gross tumor and the involved spine. PTV was produced by adding 0-2 $\mathrm{mm}$ to the CTV $[22,23]$. SBRT was applied to the lesions limited to the vertebral bodies with oligometastases, and hypofractionated RT was conducted for all other patients.

Hypofractionated RT and SBRT were planned with Eclipse version 8.6 (Varian, Palo Alto, CA, USA) and iPlan version 3.0 (Brainlab, Feldkirchen, Germany), respectively. They were performed using Varian iX (Varian) and Novalis (Brainlab), respectively. Total irradiation dose, fraction size, and the modality of RT were determined by the sites of metastatic bone lesions and adjacent normal structures.

\section{Response evaluation}

The pain response evaluation after RT was conducted by referring to the guidelines of the International Bone Metastases Consensus Working Party palliative radiotherapy endpoints [12,24]. Complete response (CR) was defined as the absence of pain after radiotherapy, partial response (PR) as a score of at least a 2 points reduction in numeric pain rating scale (NRS) scores, progressive disease (PD) as increasing NRS scores of more than 2 points, and stable disease (SD) as the status of neither PR nor PD. The radiologic response was evaluated by modified RECIST [20]. Both pain and radiologic responses were assessed using the first follow-up consultation and imaging studies within 1 to 4 months after the end of the RT.

https://doi.org/10.3857/roj.2019.00136

\section{Statistical analysis}

Survival analyses were performed with the Kaplan-Meier method, and the periods of survival were from the start of the RT for bone metastases to death or the last follow-up date. A log rank test was used to identify the survival differences in each clinical characteristic. The features that had statistically significant differences in survival were analyzed by the Cox multivariate proportional hazard regression model for multivariate analyses. All statistical analyses were carried out with SPSS version 25 (IBM Corp., Armonk, NY, USA). A p-value of less than 0.05 was considered statistically significant.

\section{Results}

\section{Clinical features}

Among the 41 patients receiving RT for bone metastases from HCC, 7 patients (17.1\%) were alive at the end of the followup. The median follow-up duration was 6.0 months (range, 0.5 to 47.0 months). The median age of the patients was 63 years old (Table 1). Of the 41 patients, 35 (85.4\%) were male, and 19 patients had an ECOG performance status 0 or 1 . Most patients (78\%) were in $\mathrm{CP}$ class $\mathrm{A}$, and 24 patients showed an elevated AFP level more than $30 \mathrm{ng} / \mathrm{mL}$. Primary tumor size in the diagnoses of the bone metastases was less than $5 \mathrm{~cm}$ in 21 patients. The primary tumor progressed in 17 patients compared with the previous tumor status.

\section{Radiotherapy}

Among 41 patients having bone metastases from HCC, 57 metastatic bone lesions were irradiated (Table 2). The spine ( $n=23,40.4 \%$ ) was the most common bony structure of metastases followed by pelvic bone ( $n=14,24.6 \%$ ). RT was planned using three-dimensional conformal radiotherapy ( $n=40,70.2 \%$ ) or intensity-modulated radiotherapy ( $=17,29.8 \%)$. Hypofractionated RT was performed in 48 lesions (84.2\%) and SBRT in 9 lesions (15.8\%). The median total prescription dose for hypofractionated RT and SBRT

Table 2. Sites of bone metastases

\begin{tabular}{lc}
\hline & No. of patients (\%) \\
\hline Spine & $24(42.1)$ \\
Pelvic bone & $13(22.8)$ \\
Rib, clavicle, scapula & $11(19.3)$ \\
Lower extremities & $4(7.0)$ \\
Skull, facial bone & $3(5.3)$ \\
Upper extremities & $2(3.5)$ \\
\hline
\end{tabular}




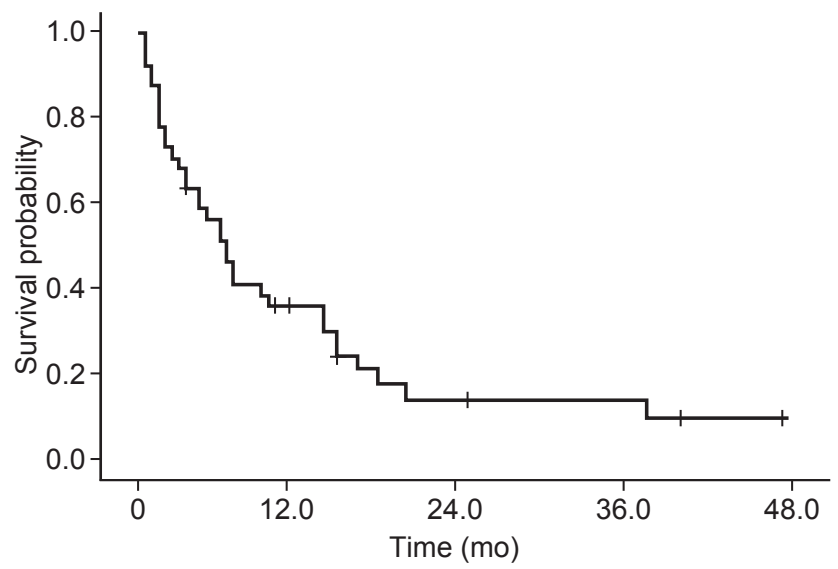

Fig. 1. A Kaplan-Meier survival curve for patients receiving palliative radiotherapy for bone metastases from hepatocellular carcinoma.

was $30 \mathrm{~Gy}$ (range, 12 to $60 \mathrm{~Gy}$ ) and $24 \mathrm{~Gy}$ (range, 21 to 27 Gy), respectively. Furthermore, the median fraction dose for hypofractionated RT and SBRT was 3 Gy (range, 3 to 4 Gy) and $8 \mathrm{~Gy}$ (range, 7 to $9 \mathrm{~Gy}$ ). After correcting the different fraction sizes with biologically equivalent dose (BED) and the $\alpha / \beta$-ratio of $10 \mathrm{~Gy}$, the median BED of the total irradiation dose was 43.2 $\mathrm{Gy}_{10}$ (range, 15.6 to $84 \mathrm{~Gy}_{10}$ ).

\section{Pain and radiologic response}

Fifty-four out of 57 metastatic lesions were assessed for pain response. CR was achieved in 3 lesions (5.6\%), and PR was achieved in 32 sites (59.3\%). The overall response rate was $64.8 \%$. The PD and SD rates were $11.1 \%$ and $24.1 \%$, respectively. For the remaining three sites without pain assessment, the patients did not complain of pain when they first came to receive RT.

Radiologic response was not available for all irradiated bone lesions. Among the 57 irradiated metastatic sites, there were 18 lesions without follow-up study due to deterioration of the patients' condition or transfer to another hospital, such as a hospice center. Thus, radiologic responses were evaluated in 39 lesions. Only one lesion showed CR (2.6\%), and 14 (35.9\%) reached PR. PD and SD were in 14 (35.9\%) and 10 (25.6\%) lesions, respectively.

\section{Survival analysis}

The median overall survival time was 6.5 months (95\% confidence interval [CI], 4.5-8.5). The 1- and 2-year survival rates after diagnosis of bone metastases were $35.5 \%$ and 13.5\%, respectively (Fig. 1). The number of patients who died

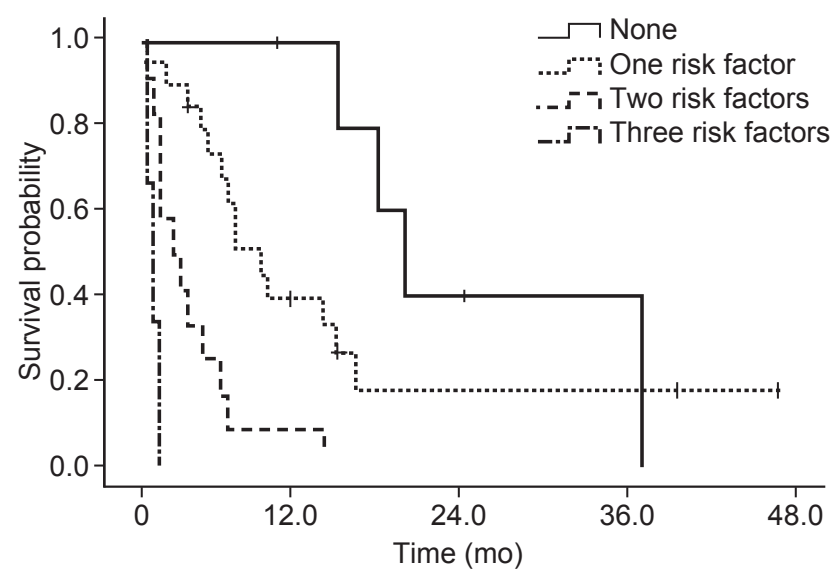

Fig. 2. Overall survival according to the number of the risk factors, which include CP class group, AFP $>30 \mathrm{ng} / \mathrm{mL}$, and primary tumor size $(p<0.001)$. CP, Child-Pugh; AFP, alphafetoprotein.

from the progression of intrahepatic and extrahepatic tumors was $9(26.5 \%)$ and $8(23.5 \%)$, respectively. Cancer-unrelated death was reported in 12 patients (35.5\%) and unknown causes in 5 (14.7\%).

Among the clinical features, the ECOG performance status group $(p=0.011), C P$ class group $(p=0.024)$, AFP $>30 \mathrm{ng} /$ $\mathrm{mL}(p=0.001)$, gamma-glutamyltransferase $(11-53 \mathrm{U} / \mathrm{L})$ level $(p=0.002)$, primary tumor size $(p=0.040)$, and primary tumor control status $(p=0.022)$ were considered statistically significant features for survival. Among these factors, the $C P$ class group $(p=0.036)$, AFP $>30 \mathrm{ng} / \mathrm{mL}(p=0.008)$, and primary tumor size $(p=0.042)$ were identified as significant risk factors from the multivariate analysis (Table 3).

\section{Triage system}

To evaluate the effect of the previously mentioned three factors on the survival, patients were divided into four groups by the number of statistically significant prognostic factors. The median survival times were $19.5(95 \% \mathrm{Cl}, 15.2-23.8), 9.0$ (95\% Cl, 5.6-12.4), 2.5 (95\% Cl, 0-5.0), and 1.0 (95\% Cl, 0.2-1.8) months for the groups with months for the groups with none, 1, 2, and 3 of the significant risk factors, respectively (Table 4). Fig. 2 shows the corresponding 6 -month survival rates of $80 \%$, $67.4 \%, 16.7 \%$, and $0 \%$ for each group, respectively.

\section{Discussion and Conclusion}

Because the diagnosis and treatment technology for HCC have improved, survival time has been prolonged. In South Korea, 
Table 3. Univariate and multivariate analyses of factors associated with survival in patients with bone metastases from hepatocellular carcinoma

\begin{tabular}{|c|c|c|c|c|}
\hline & \multicolumn{2}{|l|}{ Univariate } & \multicolumn{2}{|c|}{ Multivariate } \\
\hline & $\operatorname{HR}(95 \% \mathrm{Cl})$ & p-value & $\mathrm{HR}(95 \% \mathrm{Cl})$ & $p$-value \\
\hline Age (yr) (ref: $\leq 60)$ & & 0.291 & & \\
\hline$>60$ & $3.505(0.130-13.870)$ & & - & \\
\hline Sex (ref: male) & & 0.315 & & \\
\hline Female & $4.593(4.498-22.502)$ & & - & \\
\hline ECOG performance status (ref: 0-1) & & 0.011 & & 0.079 \\
\hline $2-4$ & $1.642(1.282-7.718)$ & & $2.372(0.928-5.796)$ & \\
\hline Child-Pugh class (ref: A) & & 0.024 & & 0.036 \\
\hline $\mathrm{B}, \mathrm{C}$ & 5.967 (3.782-10.218) & & 3.661 (1.194-11.207) & \\
\hline AFP level (ng/mL) (ref: $\leq 30)$ & & 0.001 & & 0.008 \\
\hline$>30$ & $3.570(1.629-7.371)$ & & $5.603(1.527-14.962)$ & \\
\hline ALP (ref: normal) & & 0.110 & & \\
\hline Elevated & $1.907(1.262-8.738)$ & & - & \\
\hline GGT (ref: normal) & & 0.002 & & 0.714 \\
\hline Elevated & $2.658(1.200-4.800)$ & & $1.379(0.355-5.551)$ & \\
\hline Primary tumor size $(\mathrm{cm})(\mathrm{ref}: \leq 5)$ & & 0.040 & & 0.042 \\
\hline$>5$ & $4.577(0.528-18.472)$ & & $2.768(1.223-6.267)$ & \\
\hline Primary tumor control status & & 0.022 & & 0.329 \\
\hline$C R, P R, S D$ & Ref & & Ref & \\
\hline $\mathrm{PD}$ & $1.029(0.983-5.017)$ & & $1.492(0.651-3.496)$ & 0.698 \\
\hline Unknown & $2.384(2.353-6.647)$ & & $3.211(0.981-10.791)$ & 0.137 \\
\hline PVTT (ref: no) & & 0.303 & & \\
\hline Yes & $1.465(1.342-5.658)$ & & - & \\
\hline Extra-osseous extension (ref: no) & & 0.481 & & \\
\hline Yes & $5.563(5.199-11.801)$ & & - & \\
\hline No. of bone metastasis (ref: single) & & 0.283 & & \\
\hline Multiple & $2.773(2.469-9.531)$ & & - & \\
\hline Other metastases site (ref: no) & & 0.195 & & \\
\hline Yes & $1.061(0.921-5.079)$ & & - & \\
\hline
\end{tabular}

HR, hazard ratio; $\mathrm{Cl}$, confidence interval; ECOG, Eastern Cooperative Oncology Group; AFP, alpha-fetoprotein; ALP, alkaline phosphatase; GGT, gamma-glutamyltransferase; CR, complete response; PR, partial response; SD, stable disease; PD, progressive disease; PVT, portal vein tumor thrombosis.

according to the Annual Report of Cancer Statistics, the 5-year relative survival rate was markedly increased from $10.7 \%$ to $34.6 \%$ during the last two decades, and the mortality rate also has decreased [25]. This trend has led to an increase in the frequency of distant metastases due to HCC progression because the survival duration has increased. Currently, bone metastases are one of the most common sites of extrahepatic metastasis of HCC, and bone metastases in $80 \%$ of HCC patients have been reported as the first extrahepatic sites [6]. In this situation, bone metastasis is a very important issue because it causes severe problems such as neurologic deficits as well as enormous pain, which dramatically hinders the $00 \mathrm{~L}$ of patients. RT universally alleviated pain in about 60\%-95\% of bone metastases from HCC, but the survival of the patients was heterogeneous $[12,15]$.

In the clinical setting, patients with metastatic bone lesions from HCC show very different survival periods, as reported previously $[11,13-15,17,19]$. As shown in Table 5 , the median overall survival after bone metastases was significantly different from 3-14 months. Even in other reports, wide range of survivals such as 1-48 months or 3-55 months were observed $[13,14]$. If we know the cause of the difference in the survival rate, we will be able to apply tailored treatment for the metastatic bone lesions from HCC. Therefore, identifying 
Table 4. Patient stratification according to the number of significant risk factors

\begin{tabular}{ccccc}
\hline No. of risk factors & No. of patients & Median OS (mo) & $95 \% \mathrm{Cl}$ & 6 -mo OS (\%) \\
\hline None & 6 & 19.5 & $15.2-23.8$ & 80 \\
1 & 19 & 9 & $5.6-12.4$ & 67.4 \\
2 & 12 & 2.5 & $0-5.0$ & 16.7 \\
3 & 3 & 1 & $0.2-1.8$ & 0 \\
\hline
\end{tabular}

Significant risk factors include Child-Pugh classification of B and C, alpha-fetoprotein of $>30 \mathrm{ng} / \mathrm{mL}$, and primary tumor size of $>5 \mathrm{~cm}$ $(p<0.005)$.

OS, overall survival; $\mathrm{Cl}$, confidence interval.

Table 5. Prognostic factors of HCC patients with bone metastases

\begin{tabular}{|c|c|c|c|c|}
\hline Study & Study type & Sample size & $\begin{array}{l}\text { Median OS after } \\
\text { BM (mo) }\end{array}$ & Prognostic factor \\
\hline Harding et al. [6] & Retrospective & 151 & - & AFP level, CP class, SREs \\
\hline Lu et al. [11] & Retrospective & 43 & 11 & CP class, KPS \\
\hline Chang et al. [13] & Retrospective & 102 & 3 & RT response, ECOG performance status \\
\hline Chang et al. [14] & Retrospective & 27 & 14 & Age, CP class, KPS \\
\hline He et al. [15] & Retrospective & 205 & 7.4 & $\begin{array}{l}\text { KPS, AFP level, primary HCC control, period } \\
\text { of EBRT }\end{array}$ \\
\hline Lee et al. [17] & Retrospective & 33 & 8.7 & Tomita score \\
\hline Rim et al. [18] & Retrospective & 192 & - & $\begin{array}{c}\text { ECOG performance status, primary HCC } \\
\text { control, extrahepatic metastases }\end{array}$ \\
\hline Choi and Seong [19] & Retrospective & 192 & 4.5 & $\begin{array}{l}\text { BED, performance, primary HCC control, } \\
\text { extrahepatic metastases }\end{array}$ \\
\hline Present study & Retrospective & 41 & 6.5 & $\mathrm{CP}$ class, AFP $>30 \mathrm{ng} / \mathrm{mL}$, primary tumor size \\
\hline
\end{tabular}

HCC, hepatocellular carcinoma; OS, overall survival; BM, bone metastasis; AFP, alpha-fetoprotein; CP class, Child-Pugh class; SREs, skeletal-related events; KPS, Karnofsky Performance Status scale; EBRT, external body radiotherapy; BED, biologically effective dose; ECOG, Eastern Cooperative Oncology Group; RT, radiotherapy.

prognostic factors that affect these differences in the survival rates may be helpful in setting treatment aims for patients.

In this study, CP class, AFP $>30 \mathrm{ng} / \mathrm{mL}$, and primary tumor size were found to be prognostic factors, but they were not consistent with other studies (Table 5). Harding et al. [6] performed a similar study that evaluated 151 patients with bone metastasis among 459 patients with HCC. Their results showed that the AFP level, CP class, and skeletal-related events were the statistically significant factors that affect survival, similar to the factors in this study. Several other studies presented various prognostic factors in bone metastases from HCC. Among them, the CP class and AFP level were shown to be a prognostic factor prognostic factors in three $[6,11,14]$, and two studies $[6,15]$, respectively.

In general, an increase in AFP has been reported to have a negative impact on survival in patients with $\operatorname{HCC}[26,27]$.
However, the prognostic significance of AFP was rarely mentioned in patients with bone metastases from HCC. In this study, survival was not significantly affected until the AFP reached more than $30 \mathrm{ng} / \mathrm{mL}$. Among 26 AFP elevated patients, AFP > $30 \mathrm{ng} / \mathrm{mL}$ was in 24 (92.3\%). Therefore, AFP elevation alone was not considered to represent the entire AFP elevated patients. Accordingly, we performed a comparison of survival between AFP $\leq 30 \mathrm{ng} / \mathrm{mL}$ versus AFP $>30 \mathrm{ng} / \mathrm{mL}$. The clinical implication of AFP $>30 \mathrm{ng} / \mathrm{mL}$ could in part come from a small number of patients in the present analysis.

The status of primary tumor was often reported as a prognostic factor in patients with bone metastases from HCC $[15,18,19]$. In this study, it had no statistical significance in the multivariate analysis although it was a significant factor in the log rank test. Seventeen of the 41 patients showed primary tumor progression in pre-RT evaluation, and only eight of 
them died of intrahepatic tumor progression. The remaining causes of death were three cases of extrahepatic tumor progression, four cases of cancer-unrelated causes, and two cases of unknown causes. The impact of primary tumor control status on survival was insignificant and might be affected by the small sample size. Therefore, further studies with a large patient number are needed.

Sites and pain response of bone metastases were not analyzed for survival. The reason for this was as follows. Among the 41 patients, 13 patients underwent RT for multiple bone metastatic sites simultaneously, and in 1 patient four lesions were treated during the same treatment period. Furthermore, the pain and radiologic response were not constant in patients treated for multiple sites. There was a difference in the degree of pain and radiologic response depending on the treatment site. Selection of a representative site might cause additional biases in the survival analysis. Moreover, the radiologic response analysis was limited because of the lack of follow-up imaging studies in almost one-third (31.6\%) of the total irradiated metastatic bone lesions.

To our knowledge, this is the second study that divided patients with bone metastases from HCC according to prognostic classification following Rim et al. [18]. The present study compared the survival rates according to the number of significant prognostic factors, CP class, AFP $>30 \mathrm{ng} / \mathrm{mL}$, and primary tumor size. Six-month overall survivals with no, one, two, and three prognostic factors were 80\%, 67.4\%, 16.7\%, and $0 \%$, respectively. Accordingly, we suggest that bone metastases from $\mathrm{HCC}$ with no or only one risk factor might be considered as candidates for active management.

Based on the difference in survival lengths according to the number of risk factors, we could divide them into three risk groups. For the low-risk group with no risk factor, treatment might be focused on both metastatic and primary lesions. Therefore, patients in this group could be offered active treatment for metastatic sites by surgery or SBRT, and additional care for primary HCC. Patients might benefit from relief of pain or neurologic symptoms, and prolonged survival as well. For the intermediate-risk group with only one risk factor, it is advisable to identify the primary tumor status or liver function and proceed with care accordingly. The high-risk group of two or three risk factors had only 1-2.5 months of median overall survival, and $0 \%-16.7 \%$ of 6 -month survival rate. For this group, the best supportive care for symptom palliation and hospice services should be considered, which could help relieve the patient's economic and physical burden. Therefore, shorter periods of RT might be suggested.
Disease-specific graded prognostic assessment (GPA) for spinal metastases from HCC was proposed by Rim et al. [18], based on prognostic factors such as the ECOG performance status, controlled status of primary HCC, and extrahepatic metastases other than bone. The median survival durations were 13.6, 4.8, and 2.6 months, and the 1-year overall survival rates were $58.3 \%, 17.8 \%$, and $7.3 \%$ for the lowintermediate-, and high-risk patient groups, respectively ( $p<$ 0.001) [18]. There was a difference in the prognostic factors presented, and none of the factors coincided with each other Despite these differences, GPA may provide useful information in the prognosis when making clinical decisions. Thus, the development and validation of this GPA could be a great help in determining the treatment strategy of patients.

This study has some limitations. First, this is a retrospective study. This may lead to selection bias, such as treatments other than RT, refusal of RT, etc. Additionally, it should also be noted that only patients who received radiation therapy, not the entire group of bone metastases from HCC, were included in this analysis. Second, this study had a relatively small number of patients. The numbers of the patients were not uniform when dividing the groups by clinical factors, and only a small number of patients were included in some groups even when analyzed according to the number of prognostic factors. This will require further verification through additional studies.

In conclusion, the patient classification by the number of prognostic factors showed significantly different survivals, and therefore it may help to determine a patient-specific treatment according to risk stratification. Subsequent studies will be helpful in clinical practice to distinguish which patients need either active local treatment or best supportive care.

\section{Conflict of Interest}

No potential conflict of interest relevant to this article was reported.

\section{Acknowledgments}

This work was supported by the Dong-A University research fund.

\section{References}

1. Kim BH, Park JW. Epidemiology of liver cancer in South Korea. Clin Mol Hepatol 2018;24:1-9.

2. Kim BH, Lim YS, Kim EY, et al. Temporal improvement in 
survival of patients with hepatocellular carcinoma in a hepatitis B virus-endemic population. J Gastroenterol Hepatol 2018;33:475-83.

3. Lee YT, Geer DA. Primary liver cancer: pattern of metastasis. J Surg Oncol 1987;36:26-31.

4. Toyoda $H$, Kumada $T$, Kiriyama $S$, et al. Changes in the characteristics and survival rate of hepatocellular carcinoma from 1976 to 2000: analysis of 1365 patients in a single institution in Japan. Cancer 2004;100:2415-21.

5. Ho CL, Chen S, Yeung DW, Cheng TK. Dual-tracer PET/CT imaging in evaluation of metastatic hepatocellular carcinoma. J Nucl Med 2007;48:902-9.

6. Harding JJ, Abu-Zeinah G, Chou JF, et al. Frequency, morbidity, and mortality of bone metastases in advanced hepatocellular carcinoma. J Natl Compr Canc Netw 2018;16:50-8.

7. Uchino K, Tateishi R, Shiina S, et al. Hepatocellular carcinoma with extrahepatic metastasis: clinical features and prognostic factors. Cancer 2011;117:4475-83.

8. Katyal S, Oliver JH 3rd, Peterson MS, Ferris JV, Carr BS, Baron RL. Extrahepatic metastases of hepatocellular carcinoma. Radiology 2000;216:698-703.

9. Natsuizaka M, Omura T, Akaike T, et al. Clinical features of hepatocellular carcinoma with extrahepatic metastases. J Gastroenterol Hepatol 2005;20:1781-7.

10. Fukutomi $M$, Yokota $M$, Chuman $H_{\text {, et al. Increased incidence }}$ of bone metastases in hepatocellular carcinoma. Eur J Gastroenterol Hepatol 2001;13:1083-8.

11. Lu Y, Hu JG, Lin XJ, Li XG. Bone metastases from hepatocellular carcinoma: clinical features and prognostic factors. Hepatobiliary Pancreat Dis Int 2017;16:499-505.

12. Jung IH, Yoon SM, Kwak J, et al. High-dose radiotherapy is associated with better local control of bone metastasis from hepatocellular carcinoma. Oncotarget 2017;8:15182-92.

13. Chang SS, Luo JC, Chao Y, et al. The clinical features and prognostic factors of hepatocellular carcinoma patients with spinal metastasis. Eur J Gastroenterol Hepatol 2001;13:13415.

14. Chang UK, Kim MS, Han CJ, Lee DH. Clinical result of stereotactic radiosurgery for spinal metastasis from hepatocellular carcinoma: comparison with conventional radiation therapy. J Neurooncol 2014;119:141-8.

15. He J, Zeng ZC, Tang ZY, et al. Clinical features and prognostic factors in patients with bone metastases from hepatocellular carcinoma receiving external beam radiotherapy. Cancer 2009;115:2710-20.

16. Saarto $T$, Janes $R$, Tenhunen $M$, Kouri M. Palliative radiotherapy in the treatment of skeletal metastases. Eur J Pain 2002;6:32330.

17. Lee MH, Lee SH, Kim ES, Eoh W, Chung SS, Lee CS. Survivalrelated factors of spinal metastasis with hepatocellular carcinoma in current surgical treatment modalities: a single institute experience. J Korean Neurosurg Soc 2015;58:448-53.

18. Rim CH, Choi C, Choi J, Seong J. Establishment of a diseasespecific graded prognostic assessment for hepatocellular carcinoma patients with spinal metastasis. Gut Liver 2017;11:535-42.

19. Choi C, Seong J. Predictive factors of palliative radiotherapy response and survival in patients with spinal metastases from hepatocellular carcinoma. Gut Liver 2015;9:94-102.

20. Eisenhauer EA, Therasse P, Bogaerts J, et al. New response evaluation criteria in solid tumours: revised RECIST guideline (version 1.1). Eur J Cancer 2009;45:228-47.

21. Lencioni R, Llovet JM. Modified RECIST (mRECIST) assessment for hepatocellular carcinoma. Semin Liver Dis 2010;30:52-60.

22. Ryu $S$, Jin JY, Jin $R$, et al. Partial volume tolerance of the spinal cord and complications of single-dose radiosurgery. Cancer 2007;109:628-36.

23. Ryu $S$, Yoon $H$, Stessin A, Gutman F, Rosiello A, Davis R. Contemporary treatment with radiosurgery for spine metastasis and spinal cord compression in 2015. Radiat Oncol J 2015;33:1-11.

24. Chow $E$, Hoskin P, Mitera G, et al. Update of the international consensus on palliative radiotherapy endpoints for future clinical trials in bone metastases. Int J Radiat Oncol Biol Phys 2012;82:1730-7.

25. Statistics Korea. Annual report of cancer statistics in 2016. Daejeon: Statistics Korea; 2019.

26. Tangkijvanich $P$, Anukulkarnkusol N, Suwangool $P$, et al. Clinical characteristics and prognosis of hepatocellular carcinoma: analysis based on serum alpha-fetoprotein levels. J Clin Gastroenterol 2000;31:302-8.

27. Bai DS, Zhang C, Chen $P$, Jin SJ, Jiang GO. The prognostic correlation of AFP level at diagnosis with pathological grade, progression, and survival of patients with hepatocellular carcinoma. Sci Rep 2017;7:12870. 